\title{
ENTREVISTA COM SUZANNE JILL LEVINE
}

Carolina Geaquinto Paganine- ${ }^{1}$ ${ }^{1}$ Universidade Federal Fluminense, Niterói, Rio de Janeiro, Brasil

Suzanne Jill Levine coordenou o Programa de Estudos da Tradução e foi professora de Literatura Latino-Americana e Literatura Comparada no Departamento de Espanhol e Português da Universidade da Califórnia em Santa Barbara (Estados Unidos) nos últimos trinta anos. Lá desenvolveu uma ênfase em Estudos da Tradução iniciada oficialmente em 2009 no Programa de Doutorado em Literatura Comparada, sendo que a iniciativa se expandiu recentemente para incluir uma ênfase também na graduação. Levine é uma tradutora prolífica e bastante premiada de literatura latino-americana, tendo ganhado o primeiro PEN USA West Prize de Tradução Literária (1989), o PEN American Center Career Achievement Award (1996) e uma bolsa da Fundação Guggenheim, entre outros reconhecimentos, nacionais e internacionais.

Tudo começou em 1968 quando Levine começou a explorar seu interesse pela tradução depois de realizar um intercâmbio acadêmico na Espanha como aluna da Vassar College, onde obteve o bacharelado. Na pós-graduação na Universidade de Columbia, estudou literatura latino-americana com professores como Gregory Rabassa, famoso tradutor de literatura em espanhol e português e, a partir da mentoria de Emir Rodriguez Moneal, crítico literário, pesquisador e professor de Yale, desempenhou um papel importante no boom de literatura latino-americana ao traduzir obras de Guillermo Cabrera Infante, Severo Sarduy e Manuel Puig, escritores que se tornaram conhecidos em inglês a partir do trabalho de Levine. Sua longa lista de traduções inclui obras de Adolfo Bioy 
Casares, José Donoso, Jorge Luis Borges, Julio Cortazar, Carlos Fuentes, contos e poesia de Alejandra Pizarnik e Cecilia Vicuña, entre tantos outros. Além de traduzir obras de não-ficção e alguns poemas de Borges, também atuou como editora de cinco volumes do autor argentino para a Penguin Classics (2010). Suas traduções mais recentes incluem dois livros de autores porto-riquenhos, Mundo Cruel (2013), de Luis Negron, vencedor do Lambda Prize for Fiction de 2014, e Uselessness (2017) de Eduardo Lalo, além do romance póstumo The Lizard's Tale (2012) do chileno José Donoso, vencedor do PEN USA West Award de Tradução Literária. Recentemente traduziu em colaboração com Aviva Kana, estudante de pós-graduação, The Taiga Syndrome (2018) da escritora mexicana Cristina Rivera Garza, o qual já se encontra em segunda edição. Também traduziu em colaboração dois livros da escritora argentina Silvina Ocampo, publicados no ano passado: The Promise (2019) com Jessica Powell, sua antiga aluna de pós-graduação, e Forgotten Journey (2019) com uma orientada atual, Katie Lateef-Jan. Ambos os livros foram publicados pela City Lights, a conhecida livraria e editora independente da geração "beat", situada em São Francisco. Levine é autora de livros e artigos acadêmicos sobre literatura latino-americana e tradução literária e, não menos importante, é autora da biografia literária Manuel Puig and the Spider Woman: His Life and Fictions (2000).

Levine é conhecida não só por ter traduzido escritores como os já mencionados Cabrera Infante, Sarduy e Puig, mas também por contar com a colaboração deles nas traduções, em especial Puig e Cabrera Infante. A tradutora abordou esse trabalho detalhadamente e com profundidade em seu livro The Subversive Scribe: Translating Latin American Fiction (1991), que ganhou uma segunda edição em 2009. Nessa obra, ela discorre sobre o próprio processo de tradução, tornando explícito o quanto a tarefa é também criativa e, a partir de um ponto de vista borgesiano, como as categorias de texto traduzido e original são mais complexas do que as dicotomias usuais pressupõem. Apesar de hoje as ideias pós-estruturalistas já terem sido de alguma maneira incorporadas no discurso acadêmi- 
co, certamente não era assim no início dos anos 1990 e o livro permanece provocativo e pioneiro por revelar, a partir de dentro, o que acontece no processo de traduzir ficção, um assunto subestimado e menos explorado do que a tradução de poesia. Mais ainda, The Subversive Scribe é único ao mostrar como a tarefa do tradutor é um trabalho intelectual tão válido quanto aquele do crítico literário.

Se a tarefa do tradutor é geralmente tida como invisível e subestimada, as obras e a carreira de Suzanne Jill Levine desafiam tais noções e mostram que, como Borges já havia escrito, literatura traduzida é literatura, fim de história. Ou, para usar uma expressão que aprendi com ela, Suzanne Jill Levine é "the real McCoy"1. Na seguinte entrevista, conduzida durante a minha estada na Universidade da Califórnia em Santa Barbara como Fulbright Visiting Scholar em dezembro de 2018, Levine fala de seu trabalho como tradutora e pesquisadora, mas também como biógrafa e, mas recentemente, como poeta.

Cadernos de Tradução (CT): The Subversive Scribe foi publicado primeiramente em 1991 e trata do seu trabalho desde a década de 1970 até os anos 1980 quando o boom latino-americano ainda era recente. Como foi traduzir literatura "marginal" àquela época e como é hoje em dia?

Suzanne Jill Levine (SJL): Há muitas possibilidades de resposta para essa pergunta. Primeiro, essas obras faziam parte de culturas consideradas "marginais", ou talvez "periféricas" seja um termo melhor, mas, àquela época a ênfase era nos novos escritores latino-americanos e na sua originalidade literária e, assim, usava-se termos como "experimental" ou "avant-garde". Esses novos escritores, como Cabrera Infante e Puig, chegavam pela primeira

\footnotetext{
1 De acordo com o Merriam-Webster's Unabridged Dictionary, a expressão para dizer que algo ou alguém é genuíno e autêntico, não é imitação e nem um substituto inferior.
}

Cad. Trad., Florianópolis, v. 40, n 3, p. 264-299, set-dez, 2020. 
vez ao conhecimento de um público leitor internacional. Penso que talvez um escritor "marginal" fosse ou um escritor cujo trabalho é muito inacessível ou um escritor de um país considerado periférico. Ainda usamos essas categorias hoje? É possível que a "marginalidade" fosse vista como difícil de ler, ou inacessível como no caso de Severo Sarduy que escrevia para um público restrito e não alcançou um número grande de leitores porque seus romances são mais como poesia.

(CT): Você acha que o interesse por literatura latino-americana e $o$ respectivo mercado editorial cresceram?

(SJL): Penso que agora há muito escritores desejosos por imitar o sucesso daqueles escritores de antes, ou o caráter inovador daquela geração. Houve uma proliferação de publicações e de resenhas críticas na internet e precisamos de críticos mais qualificados para discernir a qualidade da literatura contemporânea já que tantos escritores estão sendo traduzidos: há um excesso no mercado e acredito que seja difícil para os leitores identificarem o que realmente vale a pena ler. Diria que a grande diferença para mim é que quando comecei a traduzir escritores da América Latina que tinham algo de novo a trazer para a literatura, esses autores foram representados por grandes editoras como Harper \& Row, E. P. Dutton, Knopf ou New Directions. A esposa de Alfred Knopf chegou a vir ao Brasil e à Argentina para procurar escritores e, bastante cedo, travou conhecimento com Jorge Amado, por exemplo. Agora há um mercado global e um panteão de escritores internacionais, $\mathrm{Mu}$ rakami e García Márquez têm esse tipo de reputação e também Roberto Bolaño e Valerie Luiselli e uma geração mais nova talvez tenha essa reputação hoje em dia.

Mas acho que ainda hoje é difícil publicar livros, ter os livros resenhados e acho que há muito mais tradutores do espanhol para o inglês do que quando eu comecei. E o mundo editorial mudou radicalmente; essas grandes editoras tiveram que abdicar de literatura 
inovadora, ramo agora nas mãos de editoras menores como aquelas com quem tenho trabalhado ultimamente, como a Dorothy Project que faz dois livros por ano e se concentra na escrita inovadora de mulheres, como em The Taiga Syndrome; ou a Seven Stories Press, entre outras.

Vivemos em outro tempo e não percebo que há o mesmo nível de interesse ou ânimo por escritores como havia antigamente, mas isso em parte se dá porque eu estava imersa naquele mundo de escritores e agora tantas pessoas que conheço já se foram, então talvez eu não seja a melhor pessoa para responder. Converse com tradutores jovens de 30 ou 40 anos e eles poderão responder como veem o mercado hoje em dia. Ainda há escritores interessantes, mas não vejo a mesma amplitude de inovação, a sensação de que uma revolução literária está acontecendo como havia antes. Vivemos em tempos difíceis, não que sempre não tenhamos vivido em tempos difíceis - Borges tinha uma grande frase: "Como a todos los hombres le tocaron malos tiempos para vivir”. A função da literatura agora, penso eu, é muito mais social e cultural do que literária ou estética. O modo como os livros são enquadrados tem mais a ver com as questões sendo debatidas neles, como gênero e meio ambiente. A política identitária tomou conta das artes; estamos em um momento diferente.

(CT): Talvez a literatura latino-americana já tenha sido incorporada nos Estados Unidos e por isso não seja mais tão diferente ou marginal como antes, as técnicas e os temas são mais globais.

(SJL): Isso é verdade no sentido em que Susan Sontag escreveu "The World as India" e eu também diria que, por causa das novas categorias de literatura conhecidas como "latino" ou "chicano", as línguas têm se aproximado e muitos escritores latino-americanos famosos estão escrevendo em inglês. Desde os anos 1970, a língua inglesa se espalhou pelo mundo muito mais do que quando eu comecei; assim, muitos escritores querem escrever em inglês e 
muitos escritores hoje, que vivem no exílio, muitas vezes porque assim o escolheram, são mais ou menos bilíngues. Os escritores com quem eu trabalhei à época eram fora do comum, como Cabrera Infante de Cuba onde os Estados Unidos e a língua inglesa desempenhavam um papel de dominação econômica e imperialista, e Puig perdido nos Pampas escapando de um ambiente repressivo por meio de filmes americanos, e Carlos Fuentes cujo pai era diplomata e conhecia muito inglês. Esses escritores (incluindo aqueles que vinham de contextos econômicos mais modestos) tinham bastante estudo (muitas vezes autodidatas) e eram cosmopolitas. Assim, penso que o papel que a língua inglesa desempenhou em todas as transformações políticas e sociais desde os anos 1970 até agora, adentrando o novo milênio, faz com que este seja um mundo diferente. As coisas mudaram, houve uma mudança geopolítica e isso afeta a recepção e a produção literária. Os anos 1960 foram uma época especial em que os Modernistas e a Vanguarda ainda tinham uma presença forte.

(CT): Em 1962, Haroldo de Campos disse em um famoso ensaio que "Quanto mais inçado de dificuldades esse texto, mais recriável, mais sedutor enquanto possibilidade aberta de recriação" ${ }^{2}$. De modo semelhante em The Subversive Scribe você escreveu que aquilo que inicialmente a levou para a tradução foi a vontade de "tentar resolver os enigmas mais dificeis" (1991, p. vi) e, ao observarmos a sua lista de obras traduzidas, vemos facilmente uma preferência por textos "dificeis", frequentemente repletos de intertextualidade, que desafiam as fronteiras da lingua entre as modalidades escrita e oral, enquanto outros tentam expandir a língua ao serem abertamente inventivos. Você tinha, como Haroldo de Campos teve, um projeto de expandir as possibilidades criativas da língua-alvo?

${ }^{2} C f$. CAMPOS, Haroldo de. "Da tradução como criação e como crítica". In: Metalinguagem e outras metas. São Paulo: Perspectiva, 2004, p. 24.

Cad. Trad., Florianópolis, v. 40, n 3, p. 264-299, set-dez, 2020. 
(SJL): Isso é basicamente o que eu discuti em The Subversive Scri$b e$; acho que minhas ideias não mudaram tanto desde então. A razão para traduzir é talvez criar algo novo. É prestar homenagem a algo belo ou relevante que você quer que tenha uma nova vida e que seja lido por mais leitores. A sobrevivência da literatura, de um texto, é muito importante, mas você também está tentando transpor um ponto de vista, um uso da linguagem que é criativo, e isso é importante e tem algo a nos ensinar. Então sim, é a linguagem em si, obviamente, que se quer impactar, mas também se quer impactar a maneira como as pessoas pensam. Se você acha que um livro traz perspectivas diversas, isso também é relevante. Além disso, mostrar que, apesar de sermos tão diferentes, também temos coisas em comum entre línguas e culturas diversas. Em suma, penso que, como escrevi em The Subversive Scribe, é um processo e uma atividade criativa em que há uma tentativa de enriquecer a língua inglesa com aquilo que vem de outro texto. Haroldo, por sinal, foi uma pessoa que tive o prazer de conhecer. Nunca me esqueço de um jantar no domingo de Páscoa com ele e o crítico uruguaio, Emir Rodríguez Monegal, meu amigo, e Roman Jakobson e sua esposa, que eram amigos do Haroldo, e eles conversaram sobre Maiakovski, muito admirado por Haroldo, e, é claro, estar perto do Haroldo e de seu entusiasmo era muito contagiante. Como ele, me sentia intrigada pela impossibilidade de traduzir textos aparentemente intraduzíveis, como a obra de Sarduy, de quem ele também era próximo. Havia escritores forçando a linguagem para além de seus limites, num espírito joyceano. Eu não conhecia muito bem o português e ele me deu uma tradução literal do primeiro fragmento do seu livro Galáxias, e eu a traduzi: foi um desafio divertido, ele adorou as associações inventivas e os sons que eu produzi, talvez enriquecendo a leitura do texto.

(CT): Em um dos capitulos de The Subversive Scribe, você escreveu que a sua tradução de Boquitas Pintadas (1969) de Manuel Puig influenciou o autor na escrita de seu romance seguinte, The Buenos 
Aires Affair (1973), mostrando que a tradução tem o poder de funcionar em outras direções além de fonte-alvo.

SJL: Esse é outro aspecto sobre a tradução compartilhado por vários desses autores. Para Haroldo de Campos, o processo de tradução era parte de um processo criativo, tal como para Cabrera Infante tradução e escrita eram inter-relacionados. Manuel Puig também: as referências interculturais em seus livros são muito importantes, a influência da cultura de massa americana em outras culturas como a sua. $\mathrm{O}$ que Puig mostrou, enquanto estava envolvido no processo de tradução, é que a tradução causou um impacto no próximo livro que escreveria. Foi interessante ver como o que fazíamos em um livro por sua vez influenciou o próximo, um bom exemplo da possibilidade que a tradução tem de complementar o trabalho original de um escritor caso esse escritor esteja autotraduzindo ou colaborando. Agora temos uma área de pesquisa em Estudos da Tradução sobre autotradução e muitas pessoas estão escrevendo sobre isso hoje em dia. Podemos ver como esses escritores com quem eu trabalhei se tornaram paradigmáticos e é por isso que o trabalho que fiz tem importância hoje, como também o trabalho daqueles escritores latino-americanos foi de grande relevância para a cultura mundial.

(CT): Em uma entrevista anterior ${ }^{3}$, você disse que nos anos 1970 escritores frequentemente a procuravam para que traduzisse as obras deles. Isso é interessante porque parece uma inversão do movimento mais usual que é de um projeto de tradução começar pelo interesse da cultura-alvo em assimilar uma obra estrangeira. À época você estava traduzindo escritores contemporâneos que não faziam parte do cânone global e, talvez por isso também, você diria

${ }^{3}$ Cf. Levine, Suzanne Jill. Interview by María Constanza Guzmán. Words Without Borders. Out 2009. Disponível em: https://www.wordswithoutborders. org/article/an-interview-with-suzanne-jill-levine. Acesso em Nov. 2018.

Cad. Trad., Florianópolis, v. 40, n 3, p. 264-299, set-dez, 2020. 
que tinha mais espaço para criar do que se estivesse traduzindo um "clássico" da literatura?

(SJL): Acho que sim. Quando comecei a traduzir, o cenário latino-americano era dominado por grandes escritores homens, com algumas exceções como Clarice Lispector. Estamos falando de García Márquez, Mario Vargas Llosa, Cortázar, Carlos Fuentes, escritores sérios e brilhantes. Mas definitivamente eles tinham uma sensibilidade masculina e, portanto, pertencente à corrente dominante, pelo menos em questões de gênero em suas obras. Como jovem tradutora, fui cativada por escritores menos em voga, que eram subversivos de um jeito novo e intrigante. Também tive a sorte de ter a companhia de críticos literários incríveis como Emir Rodríguez Monegal, que era fascinado por artistas que iam contra as tendências do momento, como Borges o fora. Penso que havia uma fraternidade na subversividade ou, no caso dos escritores queer, talvez uma sororidade com as afinidades - e uma parte importante do que eles estavam subvertendo era o papel do autor.

(CT): Você acha que o tradutor deve ter uma certa afinidade com a obra que elelela está traduzindo? Será que a presença ou a falta de afinidade pode afetar o resultado da tradução?

(SJL): Como eu explico em The Subversive Scribe - encorajo os leitores a me lerem ali — costumo fazer projetos com os quais sinto uma conexão, então acho mesmo importante que haja algum tipo de afinidade, você deve ao menos apreciar em algum nível o que os escritores estavam fazendo e imagino que se a escrita desperta o seu interesse então o princípio do prazer faz sua parte. Não sei se isso é válido para todos os outros tradutores, mas sei que é assim comigo e, por isso, traduzir, de alguma maneira, me conduz a um espaço de alegria, de compartilhar uma sensibilidade com um colega escritor; penso que isso é parte do prazer de traduzir. 
(CT): Qual é o papel da teoria da tradução em seu trabalho como tradutora? Você pensa no seu trabalho nos termos de uma tendência domesticadora ou estrangeirizante? E como você lida com a expectativa de fluência e invisibilidade, consideradas por Lawrence Venuti como características dominantes no sistema literário dos Estados Unidos?

(SJL): Lawrence Venuti e eu éramos amigos próximos muitos anos atrás quando da publicação do meu livro e quando ele começava sua carreira como teórico da tradução. Acho que estávamos num caminho parecido àquela época quando nos conhecemos nos comitês da National Endowment for the Arts em que devíamos julgar traduções que se candidatavam a bolsas. Em um determinado momento, ele se aprofundou na teoria. Em outras palavras, eu estava mais interessada no processo de tradução em sua relação com cada texto como um ato de criação e de crítica literária. Me interessava também em articular essa relação de acordo com abordagens teóricas relevantes com uma ênfase especial na prática da tradução. Acredito que ele de fato cria regras dogmáticas sobre coisas que não se pode controlar dessa maneira. E penso que nem ele segue suas regras quando traduz. Tenho um problema com a natureza prescritiva da teoria da tradução, penso que a teoria funciona melhor numa função descritiva. Certamente, quando se é descritivo, pode-se observar "isso é domesticação" ou "esse elemento parece mais estrangeirizante". Mas dizer a alguém que faça assim... O tradutor deve fazer o que é melhor para o texto e todo texto é um mundo diferente. Depende da sua sensibilidade e de como você é sensível ao efeito que um texto está tentando produzir a partir das diversas maneiras em que a linguagem é usada e a partir da maneira em que os personagens são vistos. Acho muito difícil fazer uma regra que sirva para todos os contextos.

Com certeza há regras sobre técnicas poéticas, há formas e convenções que se um poeta está escrevendo um soneto, deve-se procurar uma forma correspondente na tradição e no uso poético em inglês. Venuti respondia, é importante observar, a um contexto cultural em par- 
ticular, isto é, à maneira como os críticos ignoravam a tradução. Acredito que ele tinha muita raiva disso, com razão, e é por isso que ele transforma aquela palavra "fluência" em uma palavra ruim porque a única coisa que um crítico diria, se dissesse qualquer coisa, era que "a tradução está bem escrita, a leitura é fluente" ou "essa tradução não é boa porque parece haver algo esquisito" e era tudo que conseguiam escrever sobre o assunto. A maioria dos críticos não tem o luxo de ter tempo ou conhecimento para levar a tradução muito a sério em seus comentários. Assim, Venuti naturalmente adotou a ideia de que "deve-se dar visibilidade" ou "veja esse processo e quem está fazendo e em que contexto?”.

Em resposta a essa questão da fluência, penso que o meu trabalho, como o de muitos tradutores que provavelmente fazem um bom trabalho, possui traços de domesticação e de estrangeirização. E é possível que isso seja verdade também para o trabalho de Venuti.

(CT): Seu trabalho como tradutora se destaca não só porque você traduziu muitos autores que, graças ao seu trabalho, são agora famosos no mundo de língua inglesa, mas também porque você escreveu sobre esse processo em seu livro. Mais recentemente, você colaborou com Luis Negrón ao traduzir Mundo Cruel para o inglês, sendo este o primeiro livro em tradução a receber o Literary Lambda Award na categoria ficção geral gay (2014) ${ }^{4}$, o que indica que o processo colaborativo pode ser bastante produtivo. Você poderia comentar esse trabalho?

(SJL): Com Luis Negrón, eu estava lidando com um livro que fora escrito com gírias de Porto Rico, em sua maioria uma gíria gay bastante específica. Eu queria, então, ser bastante precisa sobre como as palavras eram usadas, o que elas diziam e o nível de linguagem -

${ }^{4}$ Cf. "26th Annual Lambda Literary Award Finalists and Winners." Lambda Literary, 9 Jun 2014, Disponível em: www.lambdaliterary.org/26th-annuallambda-literary-award-finalists-and-winners/. Acesso em Nov. 2018.

Cad. Trad., Florianópolis, v. 40, nº 3, p. 264-299, set-dez, 2020. 
"será que isso é para dar ênfase? Será que é para ser engraçado? O que faz ser engraçado é o modo como dá a ênfase?”. Você tem que ter um domínio do registro da fala e do tom e das conotações. Foi excelente trabalhar com o Luis, como ele é de Porto Rico, um país bilíngue, ele pôde ajudar bastante e tornou o processo bem divertido. E foi incrível que pela primeira vez na história do Lambda Award para literatura gay uma tradução ganhou a categoria de ficção. Fui a coautora do Luis e ele ficou feliz com isso, por que não ficaria?

(CT): Então o que ocorre na tarefa de traduzir e colaborar que a torna mais especial, ou melhor, para além da oportunidade de consultar o autor?

SJL: A tradução é por sua própria natureza uma colaboração porque você está colaborando com o escritor original quando usa as palavras de outra pessoa para criar algo novo, mas é uma colaboração das palavras dele com as palavras que você traz. O legal é que nem sempre o autor sabe qual será a solução, mas só de praticar o diálogo já ajuda muito. Em alguns momentos você sabe a maneira como o inglês funciona, mas o colaborador consegue acrescentar conhecimento sobre o espanhol que te ajuda a compreender o máximo possível o ponto de vista do outro e como o espanhol está sendo usado. É algo simples, mas crucial que o quanto mais o autor lhe ajuda a entender o que ele ou ela estava tentando dizer em um momento específico, talvez você como tradutor encontre às vezes uma solução até melhor do que a do original. Algo parecido aconteceu com a Cristina Rivera Garza que disse ter compreendido melhor seu romance depois de lê-lo em inglês. Você é obrigada a dizer "o que estava tentando fazer aqui? O que estava tentando dizer ali?". É como dar uma segunda vida ao texto e é isso o que a colaboração faz. Vendo o processo dessa maneira, pode-se ver como é criativo e como até aprofunda o texto, mas tudo isso depende da combinação entre autor e tradutor, o quanto eles são bons para início de conversa. 
(CT): Você disse que Cabrera Infante se envolvia bastante com a tradução; e quanto à colaboração com os autores mais recentes, eles exercem um controle autoral?

(SJL): Cabrera Infante adorava fazer jogos de linguagem e introduzir aliterações e eu também fazia isso, tínhamos essa loucura em comum. Mas o que acabou acontecendo é que ele queria escrever sozinho em inglês, ele queria mesmo se apoderar do texto. Depois de alguns projetos, chegou a hora de deixá-lo continuar sozinho. De início, Cabrera Infante me deu bastante liberdade para criar soluções, mas, tenho que dizer, ao pensar se o autor exerce um controle autoral ou não, como tradutora você tem uma responsabilidade autoral. Você tenta se aproximar o máximo possível e acho que com todos eles, mais ou menos, era isso o que queriam. Então não acho que Cabrera Infante era mais controlador do que o Luis ou a Cristina, a única diferença é que ele era mais interessado no assunto porque para ele isso fazia parte de seu processo criativo.

\section{(CT): O nome dele também recebeu os créditos de tradutor.}

(SJL): Sim, Cabrera Infante queria ser considerado um escritor em inglês. Havia um pouco disso com Puig, mas ele tinha uma perspectiva diferente. Uma vez ele disse, isso é muito engraçado, em uma de suas cartas: "Eu não quero estar nos créditos da tradução porque então as pessoas vão pensar que a obra mudou”. Ele tinha um ponto de vista interessante e autoral. Disse: "Se foi traduzido por alguém, isso quer dizer que alguém fez o melhor que podia para que o livro fosse o mesmo. Mas se disser que foi o autor, aí quer dizer que o autor estava manipulando o texto e escrevendo algo diferente". É bem engraçado porque você pode olhar a coisa dos dois lados: “ah, se o autor está ali, é mais fiel” ou “ah, não, é melhor que o tradutor não esteja sendo influenciado pelo autor, que vai fazer algo diferente". Há os dois lados da coisa. Puig se 
divertia bastante com isso e o seu ego não se importava em ganhar créditos pela tradução, pelo menos não àquela época.

(CT): Em The Subversive Scribe e em outros trabalhos, você teve que justificar sua posição como tradutora mulher traduzindo em grande parte obras de escritores homens, muito embora, como você já observou, esses escritores fossem marginais ou avant-garde (muitos eram gays ou escritores exilados) ou escrevessem sobre as margens: personagens gays ou transgênero, o papel da mulher, um sociedade em desaparecimento. Como surgiu essa crítica e como você a vê hoje?

(SJL): Por que resolveram me criticar em particular e não inúmeras outras tradutoras, incluindo Edith Grossman, que fazia exatamente a mesma coisa? Penso que o motivo de eu ser criticada enquanto outras tradutoras não o eram foi porque escrevi esse livro The Subversive Scribe, em que afirmei que era subversiva; outras tradutoras tiveram o bom senso de não afirmarem que eram subversivas. Elas apenas diziam que eram tradutoras. Consigo entender o porquê das pessoas dizerem "você pode ser subversiva de um lado, mas por outro lado por que você está traduzindo escritores homens?". Tenho que admitir que a maioria das obras que traduzi era de homens, mas isso foi no início da minha carreira e, com o passar do tempo, fiz várias traduções de mulheres, inúmeras poetas e ficcionistas. Também não há dúvidas de que os homens que traduzi tendiam a ser marginais ou ter perspectivas originais e marginais, cada um a seu modo um tanto excêntrico, acredito, tanto os homens quanto as mulheres. Traduzi escritores excêntricos e penso que mais do que uma mulher, eu sou uma excêntrica.

(CT): Você acha que ainda há esse tipo de crítica ao seu trabalho e ao trabalho de outras mulheres tradutoras? 
(SJL): Sempre há uma variedade de críticas, por exemplo, recentemente houve um estardalhaço sobre uma tradução coreana em que houve críticas de que a mulher que traduziu realizou muitas mudanças como se, ingenuamente, traduzir não dissesse respeito a mudanças. Sempre haverá críticas porque a tradução, como a realidade, não é perfeita. Mas atualmente há muito mais, eu diria, reciprocidade entre homens e mulheres, homens podem ser tão marginais quanto mulheres, mulheres podem ser da corrente dominante como os homens, ao menos no campo literário e artístico. De alguma maneira, isso é diferente na esfera política, apesar de muitas de nós terem ficado deprimidas porque uma mulher não ganhou as eleições. Acredito de fato que vivemos em uma época em que mulheres escritoras são reconhecidas na América Latina e em outros lugares - muito embora nunca tenha havido somente escritores homens, mas certamente eram a maioria.

A única escritora que as pessoas conheciam nos anos 1960 da América Latina era, como já mencionado, a Clarice Lispector. Era uma voz bastante incomum, poderosa e original que foi descoberta por alguns tradutores e apresentada aos leitores. As mulheres, entretanto, em geral foram reprimidas e Virginia Woolf foi, é claro, uma grande escritora feminista que confrontou essa questão. Venho de uma época em que estávamos começando a nos rebelar seriamente: fomos a segunda onda de feminismo e depois disso veio a terceira onde que, curiosamente, às vezes anda agora para trás. No final de The Subversive Scribe, eu cito o tradutor francês Albert Bensoussan: "não importa se você é homem ou mulher, você é uma mulher quando traduz", querendo comparar o papel secundário dos tradutores e das mulheres. É um absurdo, mas é como temos vivido há séculos. Acredito que finalmente tivemos avanços, mas seriam eles precários?

(CT): Você escreveu uma importante biografia literária de Manuel Puig. O quanto da tarefa de traduzir, com seu jogo entre fidelidade e traição, ficção e realidade, pode-se perceber ao tentar colocar 
em palavras a vida de um escritor famoso que você conhecia pessoalmente? Quis são os pontos em comum entre a biografia, a crítica literária e tradução?

(SJL): Biografias são uma grande responsabilidade porque você quer que tudo esteja certo, você não quer ser incorreto com o que aconteceu em tal ano ou com quem disse o quê sobre quem. Você quer ser fiel. Em alguns aspectos, uma biografia passa por questões semelhantes à de uma tradução - as pessoas vão julgá-la contra um original, se perguntando se foi aquilo mesmo que foi dito ou "será que isso aconteceu mesmo? Está certo assim?”. Alguns irão dizer “poxa, este é um retrato incrível do Puig”, outros dirão "ah, ela tem uma visão enviesada ou um ponto de vista particular". Assim, você tem que apresentar as diferentes visões das testemunhas porque a narrativa será comparada a um "original” e outras pessoas têm uma visão daquele original que pode ser diferente da sua. É um projeto subjetivo que tem a obrigação de ser o mais objetivo possível, mas, como todas as narrativas, é uma interpretação. Fiquei na verdade bem feliz com o livro, queria colocar vários incidentes porque havia um aspecto da vida do Puig que era cheio de pessoas e eventos e viagens e eu queria representar essa agitação. E foi uma vida estressante porque ele tomava conta da família, tinha que lidar com o fato de ser gay, tentava ser bem sucedido, ele lidava com muitas questões. Algo que também foi muito interessante para mim foi que, como na tradução, foi uma maneira de praticar ser uma escritora e uma narradora, em particular a narradora de uma vida. Conversei com outros biógrafos sobre coisas como o arco de vida de uma pessoa, uma vida repleta de eventos, mas de alguma maneira há algo acontecendo nessa vida que é um tema importante ou uma obsessão, mostrando que o caráter de um homem é o seu destino, o que é uma ideia de Emerson, um de seus últimos ensaios é sobre o destino e como estamos de algum modo determinados pelo nosso caráter. Eu queria trazer à luz esse aspecto do Puig e o fato avassalador de que a sua questão principal era com o pai, tanto em um nível micro quanto macro, toda a questão da opressão patriarcal, sem mencionar a tirania persistente contra os gays. 
(CT): Você está no livro?

(SJL): Sim, um dos personagens secundários.

(CT): No seu livro de poesia, Reckoning (2012), o primeiro poema intitulado "Here I" [Aqui eu] me fez pensar na tradutora finalmente retirando a máscara, como escritora, mas ainda acompanhada de poetas e amigos, como uma introdução ao livro em si, que também traz suas traduções de diversos poetas e amigos. Quais seriam para você as diferenças entre traduzir poesia e criar os próprios poemas? E como você relaciona o ato de reconhecer [reckoning], seja a si mesma, o outro ou o passado, um tema presente nos poemas do livro, tanto à tarefa de tradução quando a de criação?

(SJL): Por que eu escrevo poesia.

Tenho brincado de escrever poesia durante grande parte da minha vida, embora nos últimos dois anos tenho tentado me dedicar à escrita criativa de não-ficção. $O$ fato de eu ter conseguido escrever alguns poemas razoáveis ou jogos poéticos de linguagem é um milagre para mim, e também o fato de ter conseguido fazer traduções razoáveis de poemas, a maioria poemas em prosa, de vários poetas, me surpreende igualmente.

Digo isso não porque não tenha um ouvido musical, mas porque não tive a disciplina de estudar prosódia de modo rigoroso ou sentar e ler, extensivamente, a poesia em língua inglesa desde Milton a Marianne Moore e além. É provável que eu tenha lido mais poetas em língua espanhola (e alguns em francês), mas, novamente, não foram muitos volumes e sim alguns poemas de um número decente de poetas e de modo não metódico.

Acredito honestamente que apenas poetastros ou leitores de poesia podem compor.

Mas acabei compondo um material suficiente para uma plaquete que tem um conteúdo bastante autobiográfico, no qual, às vezes, as traduções dos poemas são curiosamente mais autobiográficas do

Cad. Trad., Florianópolis, v. 40, no 3, p. 264-299, set-dez, 2020. 
que os meus poemas “originais”. E publiquei também uma plaquete bilíngue de um poeta da Nicarágua. Com o passar dos anos, traduzi diversos poemas, a maioria de poetas mulheres. De poetas mulheres porque sentia que as escritoras, como de costume, têm sido injustamente marginalizadas ou porque, em grande parte, sentia afinidades com as ideias, a linguagem ou as emoções na escrita delas, ou porque tinham um caráter verbal ou gráfico impressionante, como a obra de Alejandra Pizarnik, Marjorie Agosin, Eunice Odio ou Cecilia Vicuña.

Acho que sou uma escritora bem como uma tradutora, ou melhor, um verdadeiro tradutor é um escritor, apesar de às vezes bons escritores não serem bons tradutores. Escrevi alguns livros, incluindo uma longa biografia (de Manuel Puig) e seja ao brincar com um poema, seja com uma tradução, tudo faz parte da alegria (e da dor) de escrever que parece necessária a mim, que é quase um tipo de tecelaria que preciso fazer para me sentir em casa no mundo. 


\section{INTERVIEW WITH SUZANNE JILL LEVINE}

Suzanne Jill Levine directed the area of Translation Studies and taught Latin American and Comparative Literature at the Department of Spanish \& Portuguese at the University of California, Santa Barbara for the past thirty years. There she developed a PhD emphasis in Translation Studies within the Comparative Literature Program, officially inaugurated in 2009, and the program has now expanded to include a growing undergraduate minor. Levine is well known as a prolific and award-winning translator of Latin American Literature, having won the first PEN USA West Prize for Literary Translation (1989), the PEN American Center Career Achievement award (1996) and a Guggenheim Foundation fellowship, among many other honors, national and international.

It all began in 1968, when Levine began to explore her interest to translate after spending a year abroad as an undergraduate student in Spain and then receiving her B.A at Vassar College in 1967. In Graduate School at Columbia University, she studied Latin American literature. Her professors included Gregory Rabassa, the well-known translator from Spanish and Portuguese and, with the mentorship of literary critic, scholar and Yale Professor Emir Rodriguez Monegal, she came to play an important role in the Latin American Literary Boom translating works by Guillermo Cabrera Infante, Severo Sarduy and Manuel Puig, authors who became known through her words in the English speaking world. Her long list of translations includes works by Adolfo Bioy Casares, José Donoso, Jorge Luis Borges, Julio Cortazar, Carlos Fuentes, short fiction and poetry by Alejandra Pizarnik and Cecilia Vicuña and countless others. Regarding Jorge Luis Borges, in addition to translating his nonfiction and some poems, she also served recently as general editor of five paperback volumes of his work for Penguin Classics (2010). Her most recent translations include 
two Puerto Rican works, Mundo Cruel (2013) by Luis Negron, which won the 2014 Lambda Prize for Fiction, and Uselessness (2017) by Eduardo Lalo, as well as José Donoso's posthumous novel The Lizard's Tale (2012), awarded the PEN USA West Award for Literary Translation. A recent translation, in collaboration with graduate student Aviva Kana, is The Taiga Syndrome (2018) by Mexican writer Cristina Rivera Garza, now in its second printing. She has also translated in collaboration two works by the Argentinian writer Silvina Ocampo, which came out recently: The Promise (2019) with a former graduate student, Jessica Powell, and Forgotten Journey (2019) with current graduate student Katie LateefJan. Both books were published by City Lights, the well-known "beat generation" independent bookstore and publisher based in San Francisco. Levine is the author of scholarly books and essays on Latin American literature and literary translation and, last but not least, she authored the literary biography Manuel Puig and the Spider Woman: His Life and Fictions (2000).

Levine is known not only for translating writers as the aforementioned Cabrera Infante, Sarduy and Puig, but also for collaborating with them in her translations of their work, particularly Puig and Cabrera Infante. She addressed those collaborations in detail and depth in her book The Subversive Scribe: Translating Latin American Fiction (1991) which gained a new edition in 2009. Here she comments on her translating process revealing how creative the task is and, taking a Borgesian standpoint, how the categories of translation and original are more complex than the usual dichotomies presuppose. Though today those poststructuralist stances have been somewhat incorporated by literary academia, that was certainly not so in the 1990s and the book remains thought-provoking and groundbreaking for revealing from the inside what happens in the process of translating fiction, a subject underestimated and less explored than that of poetry translation. More than that, The Subversive Scribe is unique in showing how the task of the translator is an intellectual work as valid as that of the literary critic. 
If the task of the translator is generally known to be invisible and underappreciated, Suzanne Jill Levine's works and career challenge those ideas and show that, as Borges had written before, translated literature is Literature, full stop. Or, to use an idiom I learned from her, she is the real McCoy. In the following interview, conducted during my stay at UC Santa Barbara as a Fulbright Visiting Scholar in December 2018, Levine talks about her work as a translator and a scholar, but also as a biographer and a poet.

Cadernos de Tradução (CT): The Subversive Scribe was first published in 1991 and it draws on your work from 1970 through the 1980s when the Latin American Boom was still in full swing. How was it like to translate "marginal" literature at that time and how has it changed now?

Suzanne Jill Levine (SJL): This requires a many-layered answer: first, perhaps the cultures the literature came from were considered "marginal" or perhaps "peripheral" is a better term, but at that time the emphasis was on the new Latin American writers and their literary originality and terms like "experimental" or "avantgarde" were used. These new writers like Cabrera Infante and Puig were first entering the consciousness of an international reading public. I think maybe a "marginal" writer would be either a writer whose work is very inaccessible or a writer from a country considered peripheral. Do we really use such categories now? Maybe marginality was looked upon as difficult to read, or inaccessible in the case of Severo Sarduy who wrote for a narrow readership, not reaching huge audiences because his novels are more like poetry

(CT): Do you think the interest and the editorial market for Latin American Literature have grown? 
$(S J L)$ : I think that now there are a lot of writers desirous to imitate the success of those early writers, or the innovativeness of that generation; there has been a proliferation of publishing and reviewing activity on the internet; we need more and especially better critics to discern the quality of today's literature since so many different writers are being translated: there is a glut on the market, and I think it's hard for readers to figure out who is really worth reading. I would say that the big difference for me it is that when I started translating writers from Latin America who had something new to bring to literature, they were taken up by big presses like Harper \& Row, E. P. Dutton, Knopf or New Directions. The wife of Alfred Knopf actually went down to Brazil and to Argentina to look for writers and connected early on with Jorge Amado, for example. Now there is a global market and a pantheon of global writers, for example Murakami and García Márquez have that kind of reputation, Bolaño and Valerie Luiselli and a newer generation have that reputation now perhaps.

But I think it is still difficult to get books published, to get books reviewed and I think there are many more translators in English from Spanish than when I started out. And the publishing world has changed radically; those big presses have since had to give up innovative literature which belongs now in the hands of the smaller presses like the ones I've worked with recently, like the Dorothy Project which does two books a year and focuses on the innovative writing of women such as The Taiga Syndrome; or, among others, Seven Stories Press.

We live in a different time and I don't feel that there is the same level of interest or of excitement about writers as it was then, but that's partly because I was immersed in that world of writers and now so many of the people I knew are gone so I'm not perhaps the best person to consult. Talk to younger translators in their 30s and 40s who might tell you more about how they see the market nowadays. There are still interesting writers but I don't see the same breadth of innovation, the sense of a literary revolution 
going on as back then. We live in troubled times, not that we don't always live in troubled times-Borges had a great phrase "Como a todos los hombres le tocaron malos tiempos para vivir." Literature's function now, I think, is much more social and cultural than literary, or esthetic. The way books are being categorized is more in terms of the issues that they're dealing with, like gender and the environment. Identity politics took over the arts; we are in a different moment.

(CT): Maybe Latin American Literature is already kind of incorporated in the US so it's not so different or so marginal as it used to be, the techniques and the themes are more global.

$(S J L):$ That's true in the sense that Susan Sontag wrote "The World as India" and I would say also because of the new categories of Latino or Chicano literature the languages have drawn closer to each other and many famous "Latin" writers are writing in English. Since the 1970s, English has taken over the world much more than when I started out, therefore writers want to write in English and many writers today, living in exile often by choice, are bilingual, more or less. So the ones that I was working with then were unusual, like Cabrera Infante from Cuba where the U.S. \& English played a dominant economic, imperial role, and Puig lost out in the Pampas escaping a repressive environment through American movies, and Carlos Fuentes whose father was a diplomat and knew English very well. These writers (including those from modest economic backgrounds) were mostly well-educated (often self-educated) and cosmopolitan. So I think that the role English has played in all political and social transformations since the 70s up to now into the new millennium makes it a different world. Things have shifted, there has been a geopolitical shift and that affects how literature is received, how it is produced, etc. The 1960s was a particular world, where the Modernists and the Avantgarde still had a vital presence. 
(CT): In 1962, Haroldo de Campos said in a famous essay that "The more intricate the text is, the more seductive it is to 'recreate'." Similarly, you wrote in The Subversive Scribe that what initially drew you to translation was "to try to solve the most difficult puzzles" (vi) and by going through your list of translated works, we easily spot a preference for "difficult" often densely intertextual works that challenge the frontiers inside a language between written and spoken forms, while others also try to expand them by being openly inventive. Did you have, as Haroldo de Campos had, a project of expanding the target language's creative possibilities?

(SJL): Yes, that's pretty much what I discussed in The Subversive Scribe; I don't think I've changed too much from that. The reason to translate is perhaps to create something new. It's to pay homage to something beautiful or important that you want to give a new life and you want it to be read by a different reader, so the survival of literature, of a text, is very important, but you're also trying to bring across a point of view, a use of language that is creative, and that is important, that can teach us. So yes, it's the language itself, obviously, you want to impact, but also the way people think you want to impact. So, if you think a book brings some different perspectives that is also important. Also, to show, even though we're all so different, to show commonalities as well, between different cultures and languages. But basically I think, as I said in The Subversive Scribe, it's a creative process, it's a creative activity, and you're trying to enrich the English language with what is coming from this other text, that's what you're trying to do. Haroldo, by the way, is somebody who I had the joy of meeting and as a matter of fact, there was a memorable Easter Sunday dinner with him and the Uruguayan critic, Emir Rodríguez

5 Translated by Diana Gibson and Haroldo de Campos. In: Campos, Haroldo de. Novas: selected writing of Haroldo de Campos. Edited and with an introduction by A. S. Bessa and Odile Cisneros. Evanston, IL: Northwestern UP, 2007. pp. 312-326. "Quanto mais inçado de dificuldades esse texto, mais recriável, mais sedutor enquanto possibilidade aberta de recriação".

Cad. Trad., Florianópolis, v. 40, no 3, p. 264-299, set-dez, 2020. 
Monegal, my friend, and Roman Jakobson and his wife, because these were friends of Haroldo, and they talked of Mayakovski, who Haroldo admired greatly, and of course being around Haroldo and his enthusiasm was very contagious, and like him I was intrigued by the impossibility of translating what seem to be untranslatable texts, like Sarduy's, with whom he was also close. These were writers pushing language to its limits, in Joycean spirit. I knew very little Portuguese and he gave me a literal translation of the first "Galaxy" of that book Galáxias, so I did the first one: it was a fun challenge and he loved the inventive associations and sounds I brought in, perhaps enriching the reading of the text.

(CT): In one of the chapters of The Subversive Scribe, you wrote about how your translation of Manuel Puig's Boquitas Pintadas (1969) influenced the author's composition of his following novel The Buenos Aires Affair (1973), showing how the power of translation can work in other directions besides source-to-target.

(SJL): That's another aspect of translation a number of these authors shared. For Haroldo de Campos the translation process was part of the creative process, just as for Cabrera Infante translation and writing were interrelated. Manuel Puig too: the cross-cultural references in his books were very important, the influence of American popular mass culture on other cultures like his own. What Puig also showed, as he was involved in the process of the translation, that the translation impacted the next book he would write. It was interesting to see how what we did in one book then influenced the next book, a great example of the possibility that translation has to supplement the original writer's work if the original writer is self-translating or collaborating. We have now in Translation Studies an area of study about selftranslation, and people are talking about it a lot these day. You can see how these writers I worked with were paradigms, hence why the work I was doing was important for the future which is 
now, and how the work of those Latin American writers were important to world culture as well.

(CT): In a previous interview ${ }^{6}$, you said that in the 1970s writers would often approach you to translate their work. This is interesting because it seems an inversion of the usual movement of translation that begins with the target culture's interest to assimilate a foreign work. Do you think that because you were translating works from contemporary writers who were not at the time part of a global canon you were able to have more room for creation than if you were translating a "classic"?

$(S J L)$ : I think that's true. When I was starting to translate, the scene was dominated by major Latin American male writers with a few exceptions like Clarice Lispector. We're talking about García Márquez, Mario Vargas Llosa, Cortázar, Carlos Fuentes, brilliant, serious writers. But definitely their sensibility had a macho, hence mainstream side, in the sense of, at least, the gender aspect of their work. And as a young woman translating, I was drawn to less mainstream writers who were being subversive in new and interesting ways. Also I had the good fortune to be in the company of brilliant literary thinkers like Emir Rodriguez Monegal, who was fascinated by trend-breakers-as Borges had been. There was a fraternity of subversiveness, I suppose, or in the case of the queer writers, perhaps a sorority of affinity-and an important part of what they were subverting was the role of the author.

(CT): Do you think a translator has to have a kind of affinity with the work he/she is translating? Can the presence or lack of affinity affect the outcome of the translation?

6 See: Levine, Suzanne Jill. Interview by María Constanza Guzmán. Words Without Borders. Oct 2009, https://www.wordswithoutborders.org/article/aninterview-with-suzanne-jill-levine . Accessed Nov. 2018.

Cad. Trad., Florianópolis, v. 40, no 3, p. 264-299, set-dez, 2020. 
(SJL): As I explain in The Subversive Scribe-I encourage readers to read me there-I tend to do projects with which I feel a connection, so yes, I think that it is important for there to be an affinity of some sort, you have to at least appreciate in some way what they're doing and I suppose if the writing appeals to you then perhaps the pleasure principle plays its part. I don't know if this is true for all other translators, but I feel it's true for me, thus translating in a way puts me in a happy space, sharing a sensibility with a fellow writer; I think this is part of the pleasure.

(CT): What is the role of translation theory in your work as a translator? Do you consider your work in terms bent towards domestication or foreignization? And how do you deal with the expectation for fluency and invisibility, as Lawrence Venuti has claimed them to prevail in the US literary system?

(SJL): Lawrence Venuti and I were close friends many years ago, when my book was coming out and he was beginning his career as a translation theoretician, so I think that we met- actually on NEA committees where we were asked to judge translations for fellowships---on a similar path at that point. He ultimately went much further into theory. In other words, I was more interested in the process of translation and its relation to each text as an act of creation and literary criticism and in articulating that relationship according to relevant theoretical approaches with particular focus on translation practice. I do think that he is often making dogmatic rules about something you can't control in that way. And I don't even think he follows his rules when he translates. I have a problem with the prescriptive nature of translation theory, I think theory does better to serve a descriptive function. And certainly when you are descriptive you can note "yes, that's domestication" or "this element seems to be more foreignizing." But to say to somebody to do that... you have to do what's best for the text and every text is a different world. It depends on your sensibility and on how sensitive 
you are to the effect that this text is trying to produce with the way languages are being used in it, with the way characters are being seen. I think it's very hard to make a rule for all contexts.

Certainly, there are rules about poetic techniques, there are forms and conventions that if a poet is writing a sonnet then you are going to seek a correspondent form in the English poetic tradition and usage.

Venuti was responding to a particular cultural context it is important to note, that is, to the way critics ignored translation. I think he was very angry about that, and justifiably, and that's why he turns that word "fluency" into a bad word because the only thing a critic would say, if they say anything, would be "the translation reads very well, it's very fluent," or "this translation doesn't read well because it seems awkward," and that's the only thing they could come up with. Most critics really don't have the luxury of time or expertise to take translation terribly seriously in their commentaries. So, Venuti naturally adopted my idea that "you got to make it visible," or "look at this process and who is doing it and in what context?"

In response to his fluency issue, I think that my work, like most translators who probably do good work, has aspects of domestication and foreignization. And that is probably true of Venuti's work too.

(CT): Your work as a translator stands out because you not only translated many authors who, thanks to you, are now famous worldwide, but you also wrote about it in your book. More recently you collaborated with Luis Negrón in rendering Mundo Cruel into English, the first book in translation to receive a Literary Lambda Award in the General Gay Fiction category (2014) ${ }^{7}$, indicating that the collaborative process can be very productive. Could you comment on that work?

7 See "26th Annual Lambda Literary Award Finalists and Winners." Lambda Literary, 9 June 2014, www.lambdaliterary.org/26th-annual-lambda-literaryaward-finalists-and-winners/. Accessed Nov. 2018.

Cad. Trad., Florianópolis, v. 40, n⿳ 3, p. 264-299, set-dez, 2020. 
(SJL): With Luis Negrón, I was dealing with a book that was written in Puerto Rican slang, a very specific mostly gay slang, so I wanted to be very clear about how words were being used, what they were saying and the level of the language- - is this supposed to be emphatic? Is this supposed to be funny? What makes it funny is how emphatic it is?" You must have a handle on the register of speech and the tone and connotations. It was really great to work with Luis: because he comes from Puerto Rico, a bilingual country, he could be so helpful and made the process a lot of fun. And what was amazing is that for the first time in the history of the Lambda Award for gay literature, a translation won in the fiction category. I was his coauthor and he was pleased about it, why wouldn't he be?

(CT): So, what is in the work of translation and collaboration that make it special or better besides being able to consult with the author?

$(S J L)$ : Translation is by its very nature a collaboration because you are collaborating with the original author because you are using somebody else's words and you are going to create something else but it's out of the collaboration of his words and the words you bring. So what is great is that the author may not always know what the solution will be, but just playing off the dialogue is very helpful. There are ways in which you know something about the way English is, but your collaborator can add knowledge about the Spanish that helps you understand as completely as possible the other's point of view and how Spanish is being used. It is a simple but crucial thing that the more the author helps you understand what he or she was at least trying to say at a particular moment, maybe you as translator can find sometimes an even better solution than the original. Something like this happened with Cristina Rivera Garza who said that she understood her novel better after she saw it in English. You're forced to say "what are you trying to do there? What are you trying to say there?" It is like giving the text a second chance and the collaboration gives the text a second 
chance. Looking at it that way you can see how that's creative, and how it maybe even deepens the text, but all this depends on the combination of author and translator, how good the author and the translator is to begin with.

(CT): You have said that Cabrera Infante was very involved with the translation; what about these recent authors, are they very authorial?

(SJL): Cabrera Infante loved to make puns and roll out alliterations and I did too, we had that madness in common. But what happened eventually is that he wanted to write in English by himself so he really wanted to take over the text. After a few projects, it was time to let him go off on his own. Initially Cabrera Infante gave me a lot of freedom to invent solutions, but I have to say, looking at whether or not the author is authorial, you have an authorial responsibility as a translator. So you're trying to get as close as you can and I think that with all of them, more or less, that's what they want. So I don't think Cabrera Infante was more authorial than Luis or Cristina, the only difference is that he was more interested in it because for him it was part of his creative process.

(CT): His name is also in the credits as a translator.

(SJL): Yes, he wanted to be considered a writer in English. There was a little bit of that with Puig but he had a different perspective. Once he said, which is very funny, in one of his letters, "I don't want credit for the translation because then people are going to think that it is changed". He had an interesting and also authorial point of view. He said "If it's translated by a person it means somebody did the very best to make the book the same. But if it says the author, oh, it means the author was screwing around with it and doing something different". So it is very funny, you can 
look at it from both sides: "oh, if the author is there, it is more faithful" or "oh, no, but if the translator is not being influenced by the author, who's going to do something different, than it's better". So, you can look at it both ways. Puig was very funny about that and his ego was not involved with getting credit for the translation, at least at that point.

(CT): In The Subversive Scribe and elsewhere, you have had to justify your position as a woman translator translating mostly men's works, even though, as you have pointed out, these writers were marginal or avant-garde (often gay writers, or writers in exile) or they wrote about the margins: gay or transgender characters, the role of women, or a vanishing society. How did this criticism came about and how do you see it now?

$(S J L)$ : Why were they criticizing me in particular and not countless other women translators including Edie Grossman who did exactly the same thing? I guess the reason I was criticized as opposed to other women translators it is because I wrote this book The Subversive Scribe, where I said I was subversive; other women translators had the good sense not to claim they were subversive. They just said they were translators. So I can understand why people say "you may be subversive in one way, but in another way why are you translating male writers?" I've got to admit most of the books I did were by male authors, but then that was the early part of my career and as time went on I did a lot of work with women, countless poets and fiction writers who were women. There's also no doubt that the male writers I did tended to be marginal and to have original and marginal perspectives, each in his own way kind of very eccentric, I think, whether or not male or female, I did eccentric writers and I guess maybe more than a woman I'm an eccentric. 
(CT): Do you think there is still this kind of criticism of your work and the work of other women translators?

(SJL): There are always a variety of critiques, for example recently there was a fuss about a Korean translation where there was some criticism that the woman who translated it made a lot of changes as if, naively, translation weren't about changes. There is always going to be criticism because translation, like reality, is imperfect. But nowadays there is a lot more, I would say, mutuality between men and women, men can be just as marginal as women, women can be just as mainstream as men, at least in the literary and art scene. Somewhat in the political scene although so many of us were depressed by the fact that a woman didn't get voted on, or she was voted on but she didn't win the presidency. I do think that we live in a time when women writers are recognized in Latin America and elsewhere-even though it was never only male writers, but certainly the majority were. The one writer that people knew of back then in the 1960s from Latin America was, as mentioned, Clarice Lispector. A very unusual and powerful, original voice, discovered by certain translators and introduced to readers. Women, however, have been suppressed normally and Virginia Woolf was of course a great literary feminist who confronted that issue. I come from an era where we were beginning to rebel seriously: we were the second wave of feminism and then after that came the third wave who, curiously, are now sometimes going backwards.

At the end of The Subversive Scribe I cite French translator Albert Bensoussan: “it doesn't matter if you are a man or a woman, you are a woman when you translate," i.e. comparing the secondary role of translators and women. So absurd, but this is how we have been doing it for twenty centuries. I think we have finally made advances, but are they precarious?

(CT): You have written an important literary biography of Manuel Puig. How much of a translation task, with its interplay 
of faithfulness and betrayal, of fiction and reality, was involved in trying to put into words the life of a famous writer you knew personally? What are the parallels between writing a biography, literary criticism and translating?

(SJL): Biographies are a responsibility because we want to get everything right, you don't want to be inaccurate about what happened this year or about who said what to who. You want to be truthful. In some ways biography has some of the similar issues that translations have that people are going to judge it against an original, asking is that what was said or "that really happened? Is that right that way?" Some people will say "wow, that's a wonderful portrait of Puig," other people will say "oh, she has a biased or particular point of view". So you need to offer different views from witnesses because the narrative is being compared to an "original" and other people have a view of that original that may be different than yours. It's a subjective project that has the duty to be as objective as possible, but, like all narratives, it is interpretive. I was actually quite happy with the book, I wanted to get a lot of incidents in because there was a way in which his life was so crowded with people and events and travel and I wanted to represent that frenzy. And it was a stressful life because he was taking care of his family, had to deal with being gay, trying to be successful, he was dealing with so many issues. What was really interesting too for me was that, like translation, it was a way to practice being a writer and to be a narrator, in particular to be a narrator of a life. And I talked to other biographers about, for example, the arc of a person's life, a life filled with events, but somehow there is something going through this life that is an important theme or obsession, showing how character is fate, which is Emerson's idea, one of his late essays is about fate and how we are in some ways determined by our character. I wanted to bring out that aspect of Puig, and the overwhelming fact that his core problem was with the father, on both a micro and macro level, the whole question of patriarchal oppression, not to mention the persistent tyranny against gay people. 
(CT): Are you in the book?

(SJL): Yes, one of the minor characters.

(CT): In Reckoning (2012), your own book of poetry, your first poem entitled "Here I" made me think of the translator finally unmasking herself, as the writer, but still in the company of poets and friends - a kind of introduction to the book itself, which also brings forth translations of different poets and friends. How different is it to you the task of translating poetry from creating your own poems? And how do you relate the act of reckoning, be it of oneself, the other or the past, a theme that emerges from the poems in the book, both to the task of translation and creation?

$(S J L)$ : Why I write poetry.

I have played around with writing poetry for most of my life, though in the last couple of years have been trying to focus on creative non-fiction. The fact that I have managed to write a few adequate poems or wordplaypen ${ }^{8}$ poems is miraculous to me, also the fact that I have managed to compose adequate translations of poems, often prose poems, of various poets, is equally amazing to me.

I say this not because I don't have a musical ear, but because I haven't had the discipline to study prosody rigorously or to sit down and read, extensively, poetry in English from Milton to Marianne Moore and beyond. I have probably read more Spanish language (and sometimes French) poets but then again, not numerous volumes but rather here and there a few poems each by a decent number of poets, again, without any method to it. I believe that only poetasters or readers of poetry can compose it, honestly.

But I did produce enough for a chapbook that is very autobiographical in content, in which, sometimes, the translations of poems in the chapbook are curiously more autobiographical than my "original"

${ }^{8}$ A lame attempt at a portmanteau!

Cad. Trad., Florianópolis, v. 40, n⿳ 3, p. 264-299, set-dez, 2020. 
poems. And published a bilingual chapbook of a Nicaraguan poet. Over the years I have translated a number of poems, mostly by women poets. By women poets because I felt women writers, as usual, have been unjustly marginalized, or, in general, because I felt affinities with the ideas, language, or emotions in their writing or because they were verbally or graphically striking, like work by Alejandra Pizarnik, Marjorie Agosin, Eunice Odio, or Cecilia Vicuña.

I guess I am a writer as well as a translator, or rather a real translator is a writer, though sometimes good writers are not good translators. I have written some books, including a long biography (of Manuel Puig) and whether I am fiddling with a poem, or with a translation, it is all part of the joy (or pain) of writing that seems necessary to me, that is almost a kind of weaving I need to do to feel at home in the world.

\section{Referências}

Campos, Haroldo de. "Da tradução como criação e como crítica", Metalinguagem e outras metas, Campos, Haroldo de. (Org.). São Paulo: Perspectiva, 2004, p. 24.

Donoso, José. The Lizard's Tale: A Novel. Tradução de Suzanne Jul Levine. Northwestern: Northwestern University Press, 2011.

Garza, Cristina Rivera. The Taiga Syndrome. Tradução de Suzanne Jul Levine e Aviva Kana. Sheffield: And Other Stories, 2018

Lalo, Eduardo. Uselessness. Tradução de Suzanne Jul Levine. Chicago: University of Chicago Press, 2017.

Levine, Suznne Jill. The subversive scribe: translating Latin American fiction. Minneapolis: Graywolf Press, 1991.

Cad. Trad., Florianópolis, v. 40, nº 3, p. 264-299, set-dez, 2020. 
Levine, Suzanne Jill. Manuel Puig and the Spider Woman: His Life and Fictions. Wisconsin: Univ of Wisconsin Press, 2000.

Levine, Suzanne Jill. The Subversive Scribe: Translating Latin American Fiction. Champaign \& London: Dalkey Archive, 2009.

Levine, Suzanne Jill. "Interview by María Constanza Guzmán”. Words Without Borders. Out 2009. Disponível em: https://www.wordswithoutborders.org/ article/an-interview-with-suzanne-jill-levine. Acesso em Nov. 2018.

Levine, Suzanne Jill. Reckoning. Georgetown, Kentucky: Finishing Line Press, 2012.

Puig, Manuel. Boquitas pintadas. Tradução de Suzanne Jul Levine. Buenos Aires: Debolsillo, 2016.

Puig, Manuel. The Buenos Aires affair. Tradução de Suzanne Jul Levine. Chicago: Dalkey Archive Press, 2010.

Negrón, Luis. Mundo Cruel. Tradução de Suzanne Jul Levine. New York: Seven Stories Press, 2013.

Ocampo, Silvinia. La Promessa [The Promess]. Tradução de Suzanne Jul Levine e Jessica Powell. San Francisco: City Lights Publishers, 2019

Ocampo, Silvinia. Viaje olvidado [Forgotten Journey]. Tradução de Suzanne Jul Levine e Katie Lateef-Jan. San Francisco: City Lights Publishers, 2019

“26th Annual Lambda Literary Award Finalists and Winners.”. Lambda Literary, 9 Jun 2014, Disponível em: www.lambdaliterary.org/26th-annual-lambdaliterary-award-finalists-and-winners/. Acesso em Nov. 2018.

Recebido em: 04/03/2020

Aceito em: 10/06/2020

Publicado em setembro de 2020

Carolina Geaquinto Paganine. E-mail: carolinagp@id.uff.br. ORCID: https:// orcid.org/0000-0002-8958-1483. Financiamento Fulbright Brasil. 